\title{
IMPROVING THE SYSTEMS ENGINEERING DOCUMENTATION PRODUCTION PROCESS
}

\author{
Joe Kasser \\ The Anticipatory Testing Corporation \\ POB 3419 \\ Silver Spring, MD 20918 \\ Telephone 3015933316 \\ FAX 3015932624
}

\begin{abstract}
Documentation is a major component of any system. It serves the purpose of communicating who is to do (or did) what, why, when, where, and how. Document quality is a function of style, format and content. Content is application specific. The documentation process is common to the entire Systems Development Life Cycle (SDLC). Good documents can be produced at low cost with resulting benefits to both you as the writer and the organization. Good engineering documents are critical to the cost effectiveness of systems engineering. Writing good documents also important to you as a person. When you sign off on a document you are stating that the document meets your personal quality standards. Your signature on that document shows your level of competency to everyone who subsequently reads that document. Reducing the cost of preparing effective documents is an approach that can be applied in any organization and will reduce the cost of the SDLC. This paper discusses improving engineering documentation.
\end{abstract}

\section{THE PURPOSE OF A DOCUMENT}

The purpose of a document is to communicate something to someone (Kasser and Schermerhorn, 1994). For example:
- SOW. Communicates the customer's needs to the potential suppliers.

- Proposal. Communicates to the potential customer, that you understand the problem, and have the most cost effective solution.

\section{THE COST OF A DOCUMENT}

The documentation process occurs in every element of the SDLC, yet much of the documentation produced is defective. For example, requirements documents often contain vague, un-testable and unachievable requirements. Fixing defects in documentation holds the promise of reducing SDLC costs in a significant manner. As an example of the cost of defective documentation, consider just the formal and informal meetings on a typical large project resulting from trying to interpret a single defective requirements document. Now multiply the time spent in these meetings, by the number of meetings and the numbers of attendees, the unplanned labor cost of these meetings can very quickly reach $\$ 500,000$ or so over the course of a large project (Kasser 1995). Now multiply that by the number of defective documents in a large, and consider the effect on the project budget and schedule.

When most people think of the cost of a document, they think of the cost to write the 


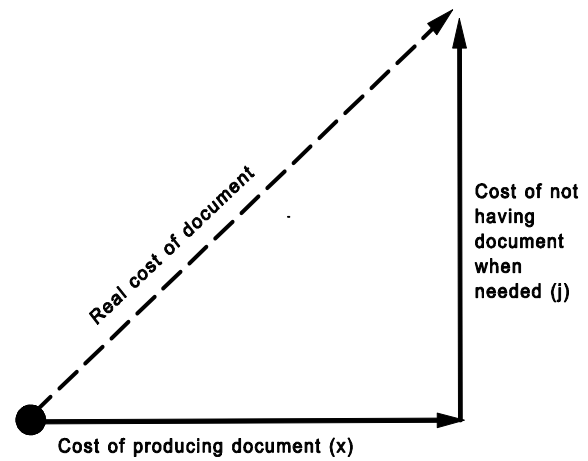

Figure 1True cost of a document

document, including the review and publish cycles. If the life cycle approach is taken then costs other than those of preparation must be taken into account when computing the cost of a document. These costs include:

- The disposal costs which are hidden in the project, because the time to empty a filing cabinet is never charged to the document being trashed, nor are the janitorial costs of disposing the document.

- The costs of using the document which are not charged to the document production process, rightly so, because the preparation phase is over.

- The costs of not having the document when it is needed. If a document is not available when needed, time and money is spent creating it.

The two elements comprising the real cost of a document are shown in Figure 1. The costs of producing the document lie on the $\mathrm{x}$ axis and the cost of not having the document when it is needed lie on the $\mathrm{j}$ axis. The true cost of the document is then given by

$$
\text { cost }=\sqrt{ }(\mathrm{x} 2+\mathrm{j} 2) \text {. }
$$

An informed trade-off between the costs of producing the document and the costs of not having the document when needed must be made when the decision is made to produce or not to produce a document (risk analysis and assessment). Examples of this technique are:
Documents which are produced and may not be needed. For example, contingency plans are Documents which might be needed. Contingency plans are produced if the cost of not having the document ready when it is needed exceeds the cost of producing the document and a probability of the contingency occurring.

Documents which are needed and not produced. Most of the work during the maintenance phase of the SDLC is adding new features or replacing aging equipment (making changes), rather than fixing bugs. Consequently, this is the phase in the SDLC where the failure to produce adequate documentation upschedule is most felt. The full effects of changes are very difficult to determine without adequate documentation (Denzler and Kasser 1995). The few dollars saved upschedule are spent several times over in trying to recreate the documents at this time, when the knowledge holders are no longer available. It can also be argued that (perceived) budget constraints prohibit producing the documents early in the SDLC even though they will be needed in the maintenance phase.

If the document is produced, there still may be costs on the $\mathrm{j}$ axis if the document is defective, i.e., if needed information is lacking (Kasser and Schermerhorn, 1994).

Reducing the costs of writing technical documentation means:

- More documents can be produced for a given budget, i.e. documents which otherwise would not be produced now can be.

- The cost to produce the required system documents will be less, resulting in lower costs for their part of the SDLC.

Documents are produced by a process. The typical real world process produces documents containing defects. Assuming that the organization uses the same finite resources in 
the typical process as it would like to use in the zero defect situation, the process can be depicted as shown in Figure 2 (Kasser 1995). When the activity begins it proceeds in a different direction away from the baseline to the checkpoint. The cost of production up to the checkpoint is the same $(\mathrm{Cm})$, but the checkpoint does not lie on the baseline anymore, rather it lies somewhere on the arc of the circle (around Point S) touching the baseline at Point T, call it Point D. The typical cost to complete is represented as a line between Point D and Point E. This cost contains two elements as shown by the arc drawn around point $\mathrm{E}$ touching the baseline at Point $\mathrm{T}$. The elements are:
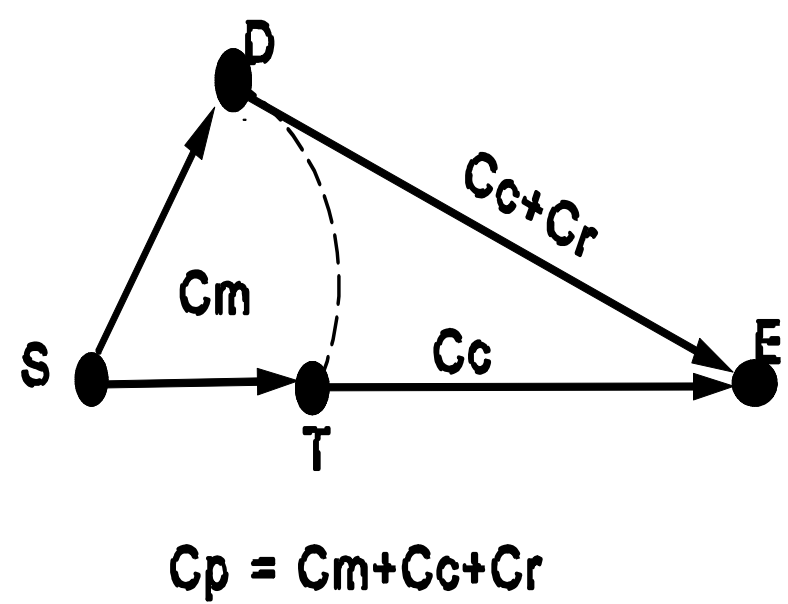

Figure 2 The cost of producing a document

- Baseline cost to complete after the checkpoint (Cc).

- Additional costs to complete due to not being on the baseline $(\mathrm{Cr})$ which represents the cost of not doing it right in the first place; costs such as scrap, inspection and rework.

In terms of the drawing in Figure 2 any activity or intermediate product that reduces the:

- deviation from the baseline;
- length of the baseline;

reduces the cost of producing the document.

Philip Crosby defines quality as conformance to specifications (Crosby 1979). By definition, if the cost to produce the (identical) product is lowered, an improvement has been made. Improvements are made by:

- Reducing the cost of the baseline process. If the cost of the process is reduced and the product still meets specifications then by definition, the process has been improved. This improvement may be implemented by making more efficient use of tools.

- Reducing the number of defects. The traditional measurement of quality is the percentage of defects introduced by the process. Reducing the number of defects using statistical process control techniques is W. Edwards Deming's approach. As number of defects gets small, the process approaches the state of doing it right the first time, i.e. Philip B. Crosby's Zero Defects approach.

\section{COMPUTER TOOLS FOR PRODUCING DOCUMENTS}

The tools used for producing documents by the majority of systems engineers today, still seem to be the basic tools (described by Eisner 1988), namely:

Word Processors. The word processor allows you to save documents and re-use sections in other documents. This is a capability which must be used sparingly, because it is very easy to insert huge sections of one document into another. Using a word processor saves time by not having to retype documents. If you add a document scanner capability to the computer, then you can scan in older documents, and reuse 
parts of them. Most word processors have spelling checkers built into them. Spelling checkers however can only tell if the spelling of the word does not exist in the english language. It does not know that you used a synonym (i.e. bear instead of bare). Outlining features are useful for scoping out documents since they can be set to provide only as much detail as necessary at a given time.

Spread Sheets. For analyzing numbers and relationships. The functions built into many spreadsheet packages provide modelling and performance analysis capabilities. All sorts of analyses may be set up in the rows and columns of the spreadsheet table, and the effect of varying the value of different parameters quickly seen. All spreadsheets have graphic display capabilities.

Graphics. For preparing drawings and presentation material.

Databases. Are useful for keeping track of requirements, names, documents, and anything else needing being kept track of.

However, in the intervening years, what began as separate packages, have evolved to use common data elements. For example, if you use compatible application packages, you can import a:

- Spreadsheet or a database as a table into a document.

- Graphics drawing as a figure into a document.

At the same time, specialized Computer Enhanced Systems Engineering (CESE) software application packages have been developed for use in specific parts of, if not throughout, the entire SDLC.

Management are familiar with the build-buy decision for the use of COTS products. They do not seem to have considered that the decision also can be made on the system engineering process to utilize personnel in a more productive manner.

Apart from their other benefits, CESE tools reduce the baseline cost of producing documentation by providing the following capabilities:

- Automate requirements extraction from Statements of Work or other source documents.

- Provide requirements traceability.

- Provide a complete history of all changes.

- Generation of documents in several standard formats.

- The ability to detect changes made in the contents of two versions of the same source document.

- A representative sample of CESE tools on display at the 4th Annual International Symposium of The National Council of Systems Engineering (NCOSE) were:

- CORE. Vitech Corporation, 2070 Chain Bridge Road, Suite 105, Vienna, VA 22182, telephone (703) 883-2270.

- The Dynamic Object Oriented Requirements System (DOORS). Zycad Corporation, Two Fountain Square, 11921 Freedom Drive, Suite 550, Reston, VA 22090, telephone (703) 904-4360.

- FORESIGHT. Nu Thena Systems, Inc., 1430 Spring Hill Road, Suite 220, McLean, VA 22102, telephone (703) 356-5056.

- Requirements Driven Development (RDD). 100. Ascent Logic Corporation, 180 Rose Orchard Way, Suite 200, San Jose, CA 95134, telephone (408) 943-0630.

- Requirements \& Traceability Management (RTM). Marconi Systems Technology, 4115 Pleasant Valley Road, Suite 100, Chantilly, VA 22021, telephone (703) 263-1260.

- SES/objectbench. Scientific and Engineering Software Inc., 4301 Westbank 
Drive, Austin, TX 78746, telephone (512) 328-5544.

- SIR/REX. Keane, Inc., 17 Computer Drive West, Albany, NY 12205, telephone (518) 453-0220.

- System Level Automation Tool for Engineers (SLATE). TD Technologies, Inc., SLATE Division, 2425 N. Central Expressway, Suite 200, Richardson, TX 75080, telephone (800) 669-4998.

- Statemate. I-Logix Inc., Three Riverside Drive, Andover, ME 01810, telephone (508) 682-2100.

\section{THE CURRENT DOCUMENTATION PREPARATION PROCESS}

While the use of the tools can reduce the cost of producing documents, they still have to be used in an effective manner. If the process is defective, the tools will just produce better defective products.

Currently, while there are guidelines as to the style and format of documents, and there are various methodologies for specifying the layout of a document, there are no precise guidelines on exactly what constitutes quality in the content of a document (Kasser 1995). Too often, the technical document preparation process takes the following form. A document is written in the form of a "brain dump". This approach results in a document:

- Written in the author's language, not that of the users.

- Containing gaps in the flow of information. These gaps are due to the detailed knowledge of the author which allows the writer to make a transition from one thought to an other, while the reader who does not have that background information is confused.

- Presenting information in an illogical order from a user's perspective.
- Containing replicated and/or redundant information.

- Containing the information the author writes, which is not necessarily the same as the information the user needs.

W. Edwards Deming stressed the importance of avoiding errors in transactions in a service industry when he wrote (Deming 1986)

"Production of an illegible figure anywhere along the line is as bad as starting off with defective material in manufacturing."

If an illegible figure is bad, a defective document is much worse. The current approach is not very effective because the document does not present the author's intent in a clear, concise and readable manner. In addition the document may be incomplete. Since it tends to fail in all categories of document metrics (Kasser and Schermerhorn 1994), some or all of the document has to be rewritten; i.e., doing the job twice.

\section{CHARACTERISTICS OF EFFECTIVE ENGINEERING DOCUMENTS}

The contents of a document have to flow from start to finish. The information has to be presented in a manner that the level of detail increases, the deeper into the document the reader delves. Where there is a danger of being bogged down in detail, the details shall be removed to an Appendix.

The document has to be formatted in a manner that much of the information can be seen when scanning it, since most people do not take the time to fully read documents. The basic rules of writing have to apply, i.e.:

- Tell the readers what you are going to tell them (introduction)

- Tell the reader (body of the text) 
- Tell the reader what you have told them (summary)

\section{THE EFFECTIVE DOCUMENT PREPARATION PROCESS}

The goals of an improved document preparation progress are to:

- Produce a useful document.

- Minimize the time spent producing the document.

The following sequence, discussed below, fits those goals:

- Locate and evaluate a similar document

- Develop metrics for the document

- Prepare an annotated outline

- Iterative part

- Produce peer review copy of document

- Circulate document for comment

- Receive comments

- Evaluate and incorporate comments

- Hold informal document review/walkthrough

- Publish draft copy of document

- Update document based on customer's comments

- Publish document

Locate and evaluate a similar document. Before you create a document, apply the lesson's learned concept from previous documents. Look for a similar document to use as basis. Examine it for good and bad points, then proceed through the document generation methodology described below. If you can't find a similar document, then reference methodologies, company standards or anything that will provide you with a first cut at the material the document has to contain.

Develop metrics for the document. Metrics for specific types of documents may be generated from Military-Standard (MIL-STD)-2167A and the set of categories for evaluating a systems description provided by (Teague and Pidgeon 1985). Their categories are:

- Completeness. The presence of all pertinent information and the lack of irrelevant and redundant information.

- Consistency. Ensuring that the terminology, style and descriptions are identical throughout a specific document and within the whole set of system documents. This category applies to graphics as well as to text.

- Correctness. The information must be correct. There are two types of errors:

- Syntax/typographical. Easy to find by means of a spelling checker or visual inspection.

- Logical. Difficult to find, since you need an understanding of the system being described in the document to know that the document is incorrect.

- Communicability. How well does the document communicate to the reader. This relates to the page layout, legibility, terminology and the use of appropriate wording.

Prepare an Annotated Outline. The usual flow in the documentation production process is to begin with an outline. This outline takes the form of a Table of Contents. The outline is agreed to, and the production of the document proceeds. However, in many instances, a Table of Contents is not good enough since it does not indicate the proposed content of each section.

The process can be improved by replacing the Table of Contents with an annotated outline, which contains the Table of Contents, together 
with a paragraph or two on each section specifying the contents of the section (Kasser and Schermerhorn 1994). This approach:

- Performs the author's brain dump in a somewhat more structured manner;

- Helps organize the document;

- Provides a cue as to what to expect in the document.

The writing process itself will only begin when the annotated outline is approved.

Before starting to write, think of the document as a presentation. The bulleted lines on the overhead transparencies are the section headings, and the written text is the verbal expansion of the bullets made by the presenter. These guidelines can be modified according to the length of the document. Use this approach to prepare a document. Plan the contents so the document flows in an orderly manner. Outline the contents using the inverted pyramid or newspaper writing style wherein the information begins with a general overview and then branches out to specific details. This way the audience can read as far as they need to. Most technical documents are scanned more often than they are read. The outlining capability of modern word processors facilitate this implementation approach because you can see the appropriate levels of detail and readily expand sections of the document as appropriate.

Prepare an annotated outline to communicate the planned contents of the document, and obtain the appropriate concurrence before starting to write the document. This approach avoids later rewrites (minimizes scrap). The following list is presented as the requirements for writing engineering documents. If a document is written once, and meets these requirements, the result will be a better document at a lower cost, due to the reduced number of changes in the review cycle (Kasser and Schermerhorn 1994).
- The information shall be written in the reader's (customer/user) language.

o The information within the document shall be pertinent to the reader.

- The information in a document shall be complete.

- All definitions shall be unambiguous.

- All information shall be well organized.

- All wording shall be clear and concise.

- Redundant or replicated information shall not be included in the document.

- All specifications or requirements shall be stated in a manner that makes them testable.

Iterative Part. Iterate as long as schedule allows:

- Review Pertinent Source Information documents. Mark or extract all pertinent information or pointers to relevant data. Build a file to refer to when creating your product.

○ Hold Informal Fact Finding Meetings. Talk with cognizant personnel who can supply:

- Source documents

- Information that is not written down.

- If you borrow documents, if you can't extract the information you need in a timely manner photocopy any pertinent information. Return the documents in a timely manner.

- Document Facts Received during Fact Finding Meetings. Document all pertinent information discussed. Send a copy to the people you spoke with and get their concurrence that the information you documented is correct. Find out where to obtain other pertinent information.

- Research Further Source Information. If your discussions identify missing information, research the subject and obtain the necessary data.

○ Discuss Write-ups in Informal Walkthroughs, Inspections and Meetings. These review sessions build the quality into the 
product during the production process. It is always cheaper to make changes to draft manuscripts than to signed-off documents. There is a tradeoff between up front costs of doing it right, and generating a draft and revising it after a meeting or review. The hardest part of creating a document is to produce the first draft. If everyone is responsible for quality then changes after the first review cycle are part of the process and not due to defects. It is easier at times to use the draft as a focus for discussion or to identify missing but needed information and ask the reviewers to supply same, as compared to spending a lot of time trying to dig out information the reviewer would have instant access to. There is an optimal point for each document, where it is cost effective to review a draft document, and incorporate changes at one time; as compared to spending more time developing the document, then holding the review and making changes.

Produce Peer Review Copy of Document. When you have gathered enough information, produce the peer review copy of the document. It doesn't have to be complete, but it does have to identify the anticipated contents of any missing sections. The peer review copy is the first informal draft of the document.

Remember most people do not read technical documents unless the really need to know the details, they tend to scan the document instead. Format the document so that pertinent information is readily seen. This paper is an example of the technique.

\section{Circulate Document for Comment.} Documents have to be checked for style, format and technical content. The technical content is best checked by a peer review process. Circulating the document at this time provides:
- Early feedback of the correctness of the information

$\circ$ Pointers to missing information

Provide copies of the document to everyone in the project (stakeholders) as well as at least one person outside the project. Project members will tend to catch errors, outside personnel will tend to note missing information.

Circulate the document to potential customers and users as well (more stakeholders). For example, circulate a requirements document to the designers who will use the document before signing off on the document. This will allow them to:

- Ensure clarification of any ambiguous elements.

- Provide an indication of missing information (something they think they will need, that is lacking from the document).

Receive Comments. There's little point in circulating a draft if you don't get any comments back. Ask for the comments by a specific date which provides enough time for people to read the document. Make it easy for busy people to make constructive comments. Provide a way to trigger their thoughts.

Think about what you'd like other people to provide you with, when they ask you to review one of their products. Ask for comments to be marked in the document in red ink (red lined). Don't require formal typed comments at this time. Provide a review form with space for specific comments as well as general comments such as:

- Great document, couldn't have done better myself

- Not bad, but still needs work in the sections listed below. 
- You left out the following points: Leave space for some points.

Evaluate and Incorporate Comments. When the comments come back, evaluate all comments and incorporate any that clarify the contents of the document. There is a good probability that some of the comments will conflict with others. Resolve these conflicts by talking with the people who wrote the comments to try to understand why they suggested their changes.

When you evaluate the comments, see if there is a pattern or trend. You may find the same type of comment occurs on several pages. Should this situation be true, you may want to rethink how you are presenting the information.

\section{Hold Informal Document Review/} Walkthrough. One good way to ensure that comments are received in a timely manner and to resolve conflicting comments is to schedule a document review meeting. The number of days If you are working with your organization's publications department:

- Agree on what they will do to your text before you authorize any work at all.

- Ensure they are cognizant of the entire process and the date you:

$\circ$ expect to provide them with the manuscript;

O need the finished document.

- Feed graphics to them according to an agreed schedule. Remember they have other things to do, and the artist may be a part time member of your team.

- Provide them with machine readable text that is compatible with their publishing software. Ensure they do not have to retype any text to minimize errors.

- If the publications department makes any errors, you will probably pay them to wait after the document is sent out, will depend on the type of document. The delay should not be too long or people will put the document aside and forget it. People should have time to review the document and mark up any comments. At the meeting, go through the document section by section and obtain consensus on changes.

Publish Formal Draft Copy of Document. This is the draft that goes to the customer for formal comments. Since you have been working closely with the customer, this stage is just a formality for contractual purposes.

Update Document Based on Customer's Comments. In the event the customer desires changes in the document, they are incorporated into the document at this time.

Publish Document. Publish the document and distribute a copy to all people with a need to know. Put a copy in the project library and send courtesy copies to outside personnel who provided information or constructive comments.

to make the errors and then correct the errors.

- Check all pages carefully, even the ones they were not supposed to touch.।

o emember it takes time to print the document, and machines tend to break down.

\section{SUMMARY}

Good engineering documents are critical to the cost effectiveness of systems engineering. Writing good documents also important to you as a person. When you sign off on a document you are stating that the document meets your personal quality standards (Kasser 1995). Your signature on that document shows your level of competency to everyone who subsequently reads that document. This paper has discussed the concepts involved in writing good documents. Learn from it and make use of it. 


\section{REFERENCES}

Crosby Philip B., Quality is Free, McGraw-Hill, 1979.

Deming, W. Edwards, Out of the Crisis, MIT

Center for Advanced Engineering Study, 1986, p 422.

Denzler David, Kasser, Joe, "Designing Budget Tolerant Systems", 5th Annual International Symposium of The National Council of Systems Engineering (NCOSE), 1995.

Eisner H., Computer Aided Systems Engineering, Prentice Hall, 1988.

Kasser Joe, Schermerhorn Robin, "Determining Metrics for Systems Engineering", 4th Annual International Symposium of The National Council of Systems Engineering (NCOSE), 1994.

Kasser, Joe, Applying Total Quality Management to Systems Engineering, Artech House, 1995.

Teague, Lavette C. Jr., Pidgeon, Christopher W., Structured Analysis Methods for Computer Information Systems, Science Research Associates, Inc., 1985, P 197.

\section{BIOGRAPHY}

Joe Kasser is president of the Anticipatory Testing Corporation, an organization he founded to reduce cost and schedule overruns in systems engineering. He has spent the last 20 years applying TQM to Systems Engineering resulting in the achievement of the cost effective implementation of international and domestic aerospace, communications and solar power systems. He is a recipient of NASA's Manned Space Flight Awareness (Silver Snoopy) Award for quality and technical excellence. He is also an Institute of Certified Professional Manager's (ICPM) Certified Manager (CM) and a recipient of the ICPM's 1993 Distinguished Service Award. Parts of this paper were developed for his proposed doctoral dissertation at The George
Washington University as well as his book on "Applying Total Quality Management to Systems Engineering". Material from the book is reproduced with permission from Artech House. 